Results 3\% of all 1289 child protection referrals seen in the 12 month period were penetrative CSA cases. $60 \%$ of cases were female and $76 \%$ were under 13 years of age. $23 \%$ of cases had another child aged 11 to 16 years as the perpetrator and $89 \%$ of these were nonrelations. $35 \%$ had previous child protection medicals performed within 3 months to 8 years. 20\% (7 cases) were forensic medical examinations and 1 of these was deemed by the author to have been seen out of the appropriate forensic sampling window. $40 \%$ of the forensic cases where emergency contraception was indicated as part of the medical care were seen more than 5 days after the incident. We performed STI screening on $82 \%$ of cases as per local protocol and of those tested there was a $6 \%$ STI rate

Conclusions A large number of penetrative CSA cases are seen yearly, many are re-referrals and a significant number are perpetrated by other children. Forensic medical examinations were indicated in $20 \%$ of cases however these often fell outside the window for timely emergency contraception and appropriate forensic sampling. A significant minority of cases seen also had an STI when screened.

We suggest particular attention should be given to timing of medical examinations to optimise not only forensic sampling but also medical care and emphasise the importance of appropriate STI screening.

\section{G219(P) AN ASSESSMENT OF THE QUALITY OF CHILD PROTECTION REPORTS CREATED AT A SINGLE NHS TRUST}

doi:10.1136/archdischild-2013-304107.231

ND Ruth, A Hughes. Paediatrics, Sandwell and West Birmingham NHS Foundation Trust, Birmingham, UK

Aim There have been a number of high profile cases around safeguarding. To compare CP medical reports to current gold standard and identify what needs improving, so that CP medical reports offer a clear opinion of the nature of the injuries identifiedThe aim of this study was to identify the gold standard for compiling safeguarding reports, and to highlight any shortfalls compared to this locally agreed standard, so that these can be improved upon for future reports.

Methods 329 child protection reports were collected over an 18-month period at a single NHS trust. Key areas assessed included documentation of: consent, date/time, any past medical/social history, growth parameters, injuries seen and location, conversations, referrals and management plans, and whether a clear opinion was given.

Results Written or verbal consent was documented in only $42 \%$ of cases. Date was documented in $97 \%$ and time in 24 hour clock in $85 \%$ of cases respectively. Reason for referral was clearly documented in $79 \%$ of cases. A background to the case was including past medical history, development, social history was poorly documented (fig.1). Cleanliness and general appearance was commented upon in $55 \%$ of cases (fig. 2). Opinions, conversations, investigations and referrals had variable reporting (fig. 3) with investigations in $152 / 329$ (46\%), treatment in $143 / 329$ (43\%), referrals $205 / 329$ $(62 \%)$ and conversations documented in $247 / 329$ (75\%) cases.

Conclusion Injuries were documented in 270/329 (82\%) cases. An interpretation of the injuries was given in 280/329 (85\%) cases, with an opinion on whether these may be consistent with the mechanism of proposed injury in 260/329 (79\%). Even though a good proportion of injuries were documented correctly and interpretation was likewise reported in $>80 \%$ of cases, this should in fact be reported in all cases and therefore although good, there is still room for improvement.

The major area of disparity was in the consent documentation which was poor. The recommendation of the study was that clear consent must always be sought and documented (including verbal consent) every time. The type and location of injury should likewise be clearly documented as well as any opinion given as to the nature of these injuries, so that there can be no ambiguity and therefore a clear judgement can be made.

\section{G220(P) PATTERNS AND MANAGEMENT OF FRACTURES IN CHILDREN UNDER 18 MONTHS IN A GENERAL HOSPITAL}

doi:10.1136/archdischild-2013-304107.232

S Gaddam Bhoomaiah, A Salim, H Grindulis. Sandwell \& West Birmingham NHS Trust, Birmingham, UK

Background The type of fracture alone cannot ascertain associated safeguarding/child protection issues, and it is therefore important to carefully assess and document each case. It is expected that all fractures under 18 months are discussed with the paediatric team in view of the raised likelihood of abusive cause in this age group.

Aims To assess whether fractures in children less than 18 months of age presenting to the emergency department (ED)are discussed with a consultant paediatrician; and to review documentation and which team(s) managed fractures in this age group.

Methods Children under 18 months of age having skeletal x-rays between September 2010-11 were identified from the Radiology IT system. For those showing a fracture information was gathered from clinical IT systems, ED notes, and hospital case notes. The type of fracture, mechanism of injury, and time to presentation were reviewed. It was determined whether the patient was discussed with a consultant paediatrician; and whether management involved the paediatric team or was solely by the ED or orthopaedic team

Results 209 x-ray reports (in 162 children) were reviewed and 55 fractures identified. Three were excluded as ED notes not found Age range was: 0-6 months 7 fractures; 6-12 months 40 and 12-18 months 8 . See table 1 for fracture types. The time interval between injury and presentation was recorded in $27(51 \%)-(74 \%<24$ hours vs. $26 \%>24$ hours). The mechanism of injury was noted in $36(70 \%)$. $21(40 \%)$ were discussed with a consultant paediatrician of whom 2 had abusive fractures. $17(33 \%)$ were referred to orthopaedics alone; and $14(27 \%)$ were managed only by ED.

Conclusions Documentation and discussion with paediatrician was inadequate. Clavicular fracture was most frequent and finger crush fractures quite common. It was not possible to judge accurately which cases were checked for social services involvement by ED. Further awareness-raising with ED staff followed by re-audit is planned.

\begin{tabular}{ll}
$\begin{array}{l}\text { Abstract G220 Table } 1 \\
\text { under 18 months }\end{array}$ & Types of fracture in children \\
\hline Clavicle 11 & \\
Radius 10 & Femur 5 \\
Ulna 4 & Tibia 7 \\
Radius + ulna 1 & Tibia +fibula 2 \\
Humerus 6 & Metatarsal 1 \\
Finger 7 & Skull 1 \\
\hline
\end{tabular}

\section{G221(P) AUDIT OF CSA (CHILD SEXUAL ABUSE) SERVICE}

doi:10.1136/archdischild-2013-304107.233

S Gopinathan. Community Paediatrics, Southwest Essex, Basildon, UK

Background In our trust the current CSA service was established in 2010 .We run weekly clinics jointly done by two experienced community paediatricians. On a monthly basis we hold a peer review meeting where cases are discussed including photographs and reports.

Details To determine whether the referral criteria are followed and to analyse the referrals received since January 2010. 